\title{
Fine structure of pentaquark multiplets in the dynamical diquark model
}

\author{
Jesse F. Giron $\odot^{1, *}$ and Richard F. Lebed $\odot^{2, \dagger}$ \\ ${ }^{1}$ Computational Physics Division (XCP), Los Alamos National Laboratory, \\ Los Alamos, New Mexico 87545, USA \\ ${ }^{2}$ Department of Physics, Arizona State University, Tempe, Arizona 85287, USA
}

(Received 18 October 2021; accepted 3 December 2021; published 22 December 2021)

\begin{abstract}
We apply the dynamical diquark model to predict the spectrum of hidden-charm pentaquark states in both unflavored and open-strange sectors. Using only Hamiltonian parameters introduced in the tetraquark $S$ - and $P$-wave multiplets, the model naturally produces the level spacing supported by the most recent LHCb results for $P_{c}$ structures. Furthermore, using model inputs obtained from data of hidden-charm, open-strange tetraquarks $\left(Z_{c s}\right)$, we predict the spectrum of $P_{c s}$ states, including the recently observed $P_{c s}(4459)$. We find all pentaquark candidates observed to date to belong to the $1 P$ multiplet and hence have positive parity.
\end{abstract}

DOI: 10.1103/PhysRevD.104.114028

\section{INTRODUCTION}

A recent burst of discoveries in the sector of heavy-quark exotic hadrons has now pushed the number of observed candidates to over 50. Multiple detailed reviews of these states have been published in recent years [1-11], but even such comprehensive reports have been unable to keep pace with the spectacular rate of new experimental findings that have occurred with remarkable regularity to the present day.

This paper focuses upon an analysis of the hiddencharm pentaquark states, labeled $P_{c}$ and $P_{c s}$ in the nonstrange and strange sectors, respectively. The first candidates $\left[P_{c}(4450), P_{c}(4380)\right]$ were observed in 2015 by the LHCb Collaboration [12] as structures in the $J / \psi p$ spectrum in the decay $\Lambda_{b} \rightarrow J / \psi p K^{-}$. Then in 2019, LHCb resolved $P_{c}(4450)$ into two peaks, $P_{c}(4440)$ and $P_{c}(4457)$, and observed a further $J / \psi p$ structure, $P_{c}(4312)$ [13]. Very recently, one more $J / \psi p$ structure $\left[P_{c}(4437)\right]$ has been observed by LHCb in the decay $B_{s}^{0} \rightarrow J / \psi p \bar{p}$ [14], but at a somewhat lower statistical significance $(>3 \sigma)$ than the other $P_{c}$ states. The only known strange candidate to date, $P_{c s}(4459)$, was also very recently observed at $\mathrm{LHCb}$ as a $J / \psi \Lambda$ structure in the decay $\Xi_{b}^{-} \rightarrow J / \psi \Lambda K^{-}$[15] (also at just over $3 \sigma$ ). The

\footnotetext{
*jgiron@lanl.gov

Richard.Lebed@asu.edu
}

Published by the American Physical Society under the terms of the Creative Commons Attribution 4.0 International license. Further distribution of this work must maintain attribution to the author(s) and the published article's title, journal citation, and DOI. Funded by SCOAP . measured masses and widths of the states are collected in Table I.

Note that all of these measurements have been made by $\mathrm{LHCb}$. The only independent evidence for $P_{c}$ states to date comes from the D0 Collaboration, which observes events consistent with the unresolved $P_{c}(4440) \rightarrow J / \psi p$ structure at $3.2 \sigma[16]$.

Much about the pentaquark states remains unknown, starting with their $J^{P}$ quantum numbers. Indeed, their parity eigenvalues alone would already reveal a great deal about their structure, since the parity of an $S$-wave $J / \psi p$ (or $J / \psi \Lambda$ ) system is -1 . The possibility of molecules composed of weakly bound $\Sigma_{c}^{(*)}-\bar{D}^{(*)}$ pairs (also in an $S$ wave) was examined even before the first $\mathrm{LHCb}$ paper [17-20]. The proximity of the $\Sigma_{c} \bar{D}^{*}$ threshold to $m_{P_{c}(4440)}$ and $m_{P_{c}(4457)}$, the $\Sigma_{c} \bar{D}$ threshold to $m_{P_{c}(4312)}$, and the $\Xi_{c} \bar{D}^{*}$ threshold to $m_{P_{c s}(4459)}$, has been noted prominently in many publications, not least of which in LHCb's own papers $[13,15]$. The relevant thresholds and $J^{P}$ values for these and related $S$-wave molecular states are conveniently tabulated in Ref. [21]. ${ }^{1}$ In particular, no $P=+$ hidden-charm pentaquark molecular thresholds occur below $4700 \mathrm{MeV}$; thus, if any of the known $P_{c}$ states is found to have $P=+$, it is not easily understood as a hadronic molecule. Since the structure $P_{c}(4380)$ is found to carry opposite parity to the original $P_{c}(4450)$ [12], then

\footnotetext{
${ }^{1}$ It should be noted that molecules in higher partial waves $(P, D, \ldots)$ are not logically precluded from forming, although the presence of a centrifugal potential barrier for $L>0$ may interfere with the binding. In addition, it seems likely that if $P$-wave molecules exist, then the lower-energy (and more prominent) $S$-wave molecules would be expected to be observed first.
} 
TABLE I. Pentaquark candidate masses and as widths measured by the LHCb Collaboration [12-15].

\begin{tabular}{lcc}
\hline \hline State & Mass $(\mathrm{MeV})$ & Width $(\mathrm{MeV})$ \\
\hline$P_{c}(4312)$ & $4311.9 \pm 0.7_{-0.6}^{+6.8}$ & $9.8 \pm 2.7_{-4.5}^{+3.7}$ \\
$P_{c}(4337)$ & $4337_{-4-2}^{+7+2}$ & $29_{-12-14}^{+26+14}$ \\
$P_{c}(4380)$ & $4380 \pm 8 \pm 29$ & $205 \pm 18 \pm 86$ \\
$P_{c}(4440)$ & $4440.3 \pm 1.3_{-4.7}^{+4.1}$ & $20.6 \pm 4.9_{-10.1}^{+8.7}$ \\
$P_{c}(4457)$ & $4457.3 \pm 0.6_{-1.7}^{+4.1}$ & $6.4 \pm 2.0_{-1.9}^{+5.7}$ \\
$P_{c s}(4459)$ & $4458.8 \pm 2.9_{-1.1}^{+4.7}$ & $17.3 \pm 6.5_{-5.7}^{+8.0}$ \\
\hline \hline
\end{tabular}

if it persists as a state, one must conclude that at least one of the $P_{c}$ 's is not molecular. ${ }^{2}$

A large body of literature has examined the $P_{c}$ states using a number of approaches: not only as hadronic molecules, but also through quark-potential models, diquark models, QCD sum rules, and others; for a discussion and numerous references, see the previously cited reviews [1-11]. Most of the relevant QCD sum-rule calculations [22-26], but not all (note Ref. [27]), postdate these reviews.

In this work, we apply a different approach, that of the dynamical diquark model, to the quantitative study of the spectroscopic fine structure of hidden-heavy-flavor pentaquarks. The dynamical diquark model is based upon the idea that multiquark systems occasionally form configurations in which the attraction of two quarks in a color-triplet channel is greater than that of either quark to the nearest antiquark in a color-singlet channel. One may then describe the full hadron in terms of compounds of diquark $\left[\delta \equiv(Q q)_{\overline{3}}\right][28]$ and triquark $\left[\bar{\theta} \equiv\left(\bar{Q}_{\overline{\mathbf{3}}}\left(q_{1} q_{2}\right)_{\overline{\mathbf{3}}}\right)_{\mathbf{3}}\right]$ [29] quasiparticles. In order for this organization to be sensible, the quasiparticles must achieve sufficient spatial separation to be described as interacting through a potential $V(r)$. If each color-triplet quasiparticle component carries at least one heavy quark (labeled above as $Q, \bar{Q})$, then $V(r)$ may be modeled using the same static potentials as the ones that are calculated in lattice simulations of quarkonium and its hybrid excitations, leading to a description of exotic hadrons in terms of the Born-Oppenheimer (BO) approximation. This dynamical diquark model and the spectroscopy of tetraquark and pentaquark states obtained from it were first described in Ref. [30].

With a predictive model in hand, it becomes possible to study the multiplet band structure for $c \bar{c} q \bar{q}^{\prime}$ and $c \bar{c} q q q$

\footnotetext{
${ }^{2}$ Other issues with molecular interpretations of $P_{c}$ involve the $J^{P}$ of the particle responsible for binding. In the case of $P_{c}(4312)$, a $0^{-}$meson like $D$ cannot support a trilinear coupling to another $0^{-}$meson like $\pi$ or $\eta$; at minimum, a $\rho$-like exchange would be necessary. And if nonstandard meson coupling fields like $\rho$ are sufficient for binding hadronic molecules, then one might expect states, bound by other light mesons, that include the isoscalar $\Lambda_{c}$ to have appeared in the existing data.
}

states numerically, as was first done in Ref. [31]. The fine structure of the ground-state ( $S$-wave) and first excitedstate $(P$-wave) tetraquark multiplets were first studied in Refs. [32,33], respectively. The application to $b \bar{b} q \bar{q}^{\prime}$ and $c \bar{c} s \bar{s}$ states appears in Ref. [34], $c \bar{c} c \bar{c}$ states in Ref. [35], and $c \bar{c} q \bar{s}$ states in Ref. [36].

However, the fine structure of the hidden-charm pentaquark states has not yet been analyzed in this model, largely due to the absence of any particular $P_{c}$ state for which the $J^{P}$ quantum numbers are definitively known. In all the tetraquark cases, a specific state [e.g., $X(3872), Z_{b}(10610)$, and $X(4140)]$ can be identified as the cornerstone upon which the rest of the multiplet is built, but the pentaquark sector to date lacks such a candidate. However, one expects that the same fine-structure Hamiltonian applies to the tetraquark $\delta-\bar{\delta}$ and pentaquark $\delta-\bar{\theta}$ sectors, and moreover, that comparing the nonstrange $c \bar{c} q \bar{q}^{\prime}$ and strange $c \bar{c} q \bar{s}$ tetraquarks allows one to determine properties of the $P_{c s}$ states from the corresponding $P_{c}$ states [36].

The observation of the new $P_{c}(4337)$ state produces a very interesting spectrum (see Table I): Two narrow, closely spaced pairs of states $\left[P_{c}(4312), P_{c}(4337)\right.$, and $P_{c}(4440)$, $\left.P_{c}(4457)\right]$ with nearly the same mass splitting. The dynamical diquark model, as shown in this paper, produces a unique combination of such states in its pentaquark spectrum: The lower pair are $J^{P}=\frac{1}{2}+$, and the upper pair are $J^{P}=\frac{3}{2}^{+}$. Since all of these are $Q \bar{Q} q q q$ states with $P=+$, they are $P$-wave states; we predict masses for the other states in this multiplet, as well as those in the lower $S$-wave multiplet.

We further use these results, as discussed above, to predict the masses of the corresponding $P_{c s}$ states. In this case, the crucial ingredient of the analysis is a comparison [36] of the well-known hidden-charm nonstrange states and the newly observed open-strange, hidden-charm tetraquark states $Z_{c s}$ :

$$
\begin{aligned}
m_{Z_{c s}(3985)} & =3982.5_{-2.6}^{+1.8} \pm 2.1 \mathrm{MeV}, \\
\Gamma_{Z_{c s}(3985)} & =12.8_{-4.4}^{+5.3} \pm 3.0 \mathrm{MeV},
\end{aligned}
$$

from the BESIII Collaboration [37] in the process $e^{+} e^{-} \rightarrow K^{+}\left(D_{s}^{-} D^{* 0}+D_{s}^{*-} D^{0}\right)$, and

$$
\begin{aligned}
m_{Z_{c s}(4000)} & =4003 \pm 6_{-14}^{+4} \mathrm{MeV}, \\
\Gamma_{Z_{c s}(4000)} & =131 \pm 15 \pm 26 \mathrm{MeV}, \\
m_{Z_{c s}(4220)} & =4216 \pm 24_{-30}^{+43} \mathrm{MeV}, \\
\Gamma_{Z_{c s}(4220)} & =233 \pm 52_{-73}^{+97} \mathrm{MeV},
\end{aligned}
$$

from the $\mathrm{LHCb}$ Collaboration in the process $B^{+} \rightarrow$ $\phi\left(J / \psi K^{+}\right)$[38].

Other diquark-based pentaquark models for have appeared in the literature. For example, Ref. [39] was based upon the diquark-triquark proposal of Ref. [29], 
its model containing spin-dependent, but not flavordependent, couplings. The model of Ref. [40] is also of diquark-triquark type, but both of the heavy quarks $c \bar{c}$ reside in the triquark. The very recent Ref. [41] appeared subsequent to the discovery of $P_{c s}(4459)$, but is a diquarkdiquark-antiquark model. This work is the first one to treat $P_{c}(4337)$ as a diquark-based state.

This paper is organized as follows: In Sec. II, we reprise the notation of the model for identifying the pentaquark states within their BO multiplets. Section III introduces the mass Hamiltonian for $S$-wave and $P$-wave multiplets, and presents expressions for the masses of all their component states. In Sec. IV, we analyze these expressions numerically, using inputs from our previous work in the tetraquark sector, and predict values for all unknown $S$ - and $P$-wave hidden-charm pentquarks in both light and open-strange sectors. Section V presents our conclusions.

\section{STATES OF THE MODEL}

A cataloguing of $Q \bar{Q} q q_{1} q_{2}$ (and $Q \bar{Q} q_{1} \bar{q}_{2}$ ) states in the dynamical diquark model, where $q \in\{u, d, s\}$ and $q_{i} \in\{u, d\}$, first appears in Ref. [30]. The same notation, with small modifications, is applied to $c \bar{c} s \bar{s}$ in Ref. [34] and to $c \bar{c} c \bar{c}$ in Ref. [35]. All confirmed exotic candidates to date have successfully been accommodated within the lowest $\left(\Sigma^{+}\right)$Born-Oppenheimer (BO) potential of the gluon field connecting the heavy diquark $[\delta \equiv(Q q)]$-triquark $\left[\bar{\theta} \equiv\left(\bar{Q}\left(q_{1} q_{2}\right)\right)\right]$ quasiparticles. The pentaquark BO potentials lack one discrete quantum number $(g, u)$ compared to those for $\delta-\bar{\delta}$ tetraquarks [30]. In all cases, $\delta, \bar{\theta}$ are assumed to transform as color triplets (or antitriplets), and each quasiparticle contains no internal orbital angular momentum.

In the case of $Q \bar{Q} q q_{1} q_{2}$, the classification scheme then begins with three possible core states in which the $\delta-\bar{\theta}$ pair lie in a relative $S$ wave. The most minimal form of the model also restricts to states in which the diquark $\delta^{\prime}=\left(q_{1} q_{2}\right)$ internal to $\bar{\theta}$, consisting of only light $u, d$ quarks, is a "good" diquark: isosinglet and $s_{\delta^{\prime}}=0$, which is expected to be the most tightly bound combination from light-hadron phenomenology [42] and from lattice simulations [43]. In principle, the "bad" (isotriplet, $s_{\delta^{\prime}}=1$ ) combination could also appear, but if so would form pentaquark states substantially higher in mass. Such states may be so broad as to have escaped detection at LHCb. Indicating the total spin $s$ of $\delta, \bar{\theta}$ by $s_{\delta}, s_{\bar{\theta}}$, respectively, and including a subscript on the full state to indicate its total spin, one obtains the spectrum

$$
\begin{aligned}
& J^{P}=\frac{1^{-}}{2}: P_{\frac{1}{2}} \equiv\left|\frac{1}{2}{ }_{\bar{\theta}}, 0_{\delta}\right\rangle_{\frac{1}{2}}, \quad P_{\frac{1}{2}}^{\prime} \equiv\left|\frac{1}{2}, 1_{\delta}\right\rangle_{\frac{1}{2}}, \\
& J^{P}=\frac{3^{-}}{2}: P_{\frac{3}{2}} \equiv\left|\frac{1}{2}, 1_{\delta}\right\rangle_{\frac{3}{2}},
\end{aligned}
$$

where again, the diquark $\delta^{\prime}$ spin $s_{\delta^{\prime}}$ is fixed to zero, and so the triquark $\bar{\theta}$ spin $s_{\bar{\theta}}=\frac{1}{2}$. Since four quark angular momenta $\left(s_{q}, s_{\delta^{\prime}}, s_{Q}, s_{\bar{Q}}\right)$ are combined here, one may transform these states into other convenient bases by means of $9 j$ angular momentum recoupling coefficients. In particular, in the basis of good total heavy-quark $(Q \bar{Q})$ and all-light baryonic $\left(B \equiv q \delta^{\prime}=q q_{1} q_{2}\right)$ spin, the transformation reads

$$
\begin{array}{r}
\left\langle\left(s_{q} s_{\delta^{\prime}}\right) s_{B},\left(s_{Q} s_{\bar{Q}}\right) s_{Q \bar{Q}}, S \mid\left(s_{q} s_{Q}\right) s_{\delta},\left(s_{\bar{q}} s_{\bar{Q}}\right) s_{\bar{\theta}}, S\right\rangle \\
=\left(\left[s_{B}\right]\left[s_{Q \bar{Q}}\right]\left[s_{\delta}\right]\left[s_{\bar{\theta}}\right]\right)^{1 / 2}\left\{\begin{array}{ccc}
s_{q} & s_{\delta^{\prime}} & s_{B} \\
s_{Q} & s_{\bar{Q}} & s_{Q \bar{Q}} \\
s_{\delta} & s_{\bar{\theta}} & S
\end{array}\right\},
\end{array}
$$

using the abbreviation $[s] \equiv 2 s+1$ for the multiplicity of a spin- $s$ state here and below. Combining Eqs. (3) and (4), one then obtains

$$
\begin{aligned}
J^{P}=\frac{1-}{2}: P_{\frac{1}{2}} & =-\frac{1}{2}\left|\frac{1}{2} B, 0_{Q \bar{Q}}\right\rangle_{\frac{1}{2}}+\frac{\sqrt{3}}{2}\left|\frac{1}{2} B, 1_{Q \bar{Q}}\right\rangle_{\frac{1}{2}}, \\
P_{\frac{1}{2}}^{\prime} & =\frac{\sqrt{3}}{2}\left|\frac{1}{2} B, 0_{Q \bar{Q}}\right\rangle_{\frac{1}{2}}+\frac{1}{2}\left|\frac{1}{2}{ }^{B}, 1_{Q \bar{Q}}\right\rangle_{\frac{1}{2}}, \\
J^{P} & =\frac{3}{2}^{-}: P_{\frac{3}{2}}=\left|\frac{1}{2} B, 1_{Q \bar{Q}}\right\rangle_{\frac{3}{2}} .
\end{aligned}
$$

In this work, it is especially convenient to employ a basis of states carrying a unique value of $s_{Q \bar{Q}}$ and of $s_{B}$ (the latter always being $\frac{1}{2}$ since $s_{\delta^{\prime}}=0$ and $s_{q}=\frac{1}{2}$ ). These states are the combinations

$$
\begin{aligned}
& \tilde{P}_{\frac{1}{2}} \equiv\left|\frac{1}{2} B, 0_{Q \bar{Q}}\right\rangle_{\frac{1}{2}}=-\frac{1}{2} P_{\frac{1}{2}}+\frac{\sqrt{3}}{2} P_{\frac{1}{2}}^{\prime}, \\
& \tilde{P}_{\frac{1}{2}}^{\prime} \equiv\left|\frac{1}{2} B, 1_{Q \bar{Q}}\right\rangle_{\frac{1}{2}}=+\frac{\sqrt{3}}{2} P_{\frac{1}{2}}+\frac{1}{2} P_{\frac{1}{2}}^{\prime}, \\
& P_{\frac{3}{2}}=\left|\frac{1}{2} B, 1_{Q \bar{Q}}\right\rangle_{\frac{3}{2}} .
\end{aligned}
$$

The $Q \bar{Q} q q_{1} q_{2}$ states in the multiplets $\Sigma^{+}(1 S)$ and $\Sigma^{+}(1 P)$ are sufficient to accommodate all particles considered in this work. However, we note that Ref. [30] also provides a classification of higher radial and orbital excitations [such as $\Sigma^{+}(n D)$ ], as well as states in excited-glue BO potentials such as $\Pi^{+}$(which are exotic analogues to hybrid hadrons). The corresponding $Q \bar{Q} q_{1} \bar{q}_{2}$ states in the $\Sigma_{g}^{+}(1 S)$ and $\Sigma_{g}^{+}(1 P)$ multiplets were first studied in Refs. [32,33], respectively, while radiative transitions between them are computed in Ref. [44]. 


\section{PENTAQUARK MASS HAMILTONIAN}

\section{A. $S$-wave Hamiltonian and mass expressions}

The $\Sigma^{+}(1 S)$ (ground-state) pentaquark multiplet contains only three states, those listed in Eqs. (3) or (6). For this multiplet, the Hamiltonian closely follows the form used for the $\Sigma_{g}^{+}(1 S)$ tetraquark multiplet in Ref. [32], and reads

$$
\begin{aligned}
H= & M_{0}+\Delta H_{\kappa_{q Q}}+\Delta H_{\kappa_{\delta^{\prime}}}+\Delta H_{V_{8}} \\
= & M_{0}+2\left[\kappa_{q Q}\left(\vec{s}_{q} \cdot \vec{s}_{Q}\right)+\kappa_{\delta^{\prime}}\left(\vec{s}_{\delta^{\prime}} \cdot \vec{s}_{\bar{Q}}\right)\right] \\
& +V_{8}\left(\lambda_{a}^{8} \vec{\sigma}_{\delta^{\prime}}\right) \cdot\left(\lambda_{a}^{8} \vec{\sigma}_{q}\right) .
\end{aligned}
$$

Since the diquark $\delta^{\prime}$ is isoscalar by assumption, this Hamiltonian lacks the nontrivial isospin dependence of its tetraquark analogue. Under the assumption that $\delta^{\prime}$ maintains its flavor content (i.e., does not exchange quarks with the other diquark), then $\pi$-like and $K$-like exchanges do not occur. ${ }^{3}$

Its matrix elements are

$$
\begin{aligned}
M= & M_{0}+\frac{\kappa_{q Q}}{2}\left[2 s_{\delta}\left(s_{\delta}+1\right)-3\right]+\frac{\kappa_{\delta^{\prime} \bar{Q}}}{4}\left[4 s_{\bar{\theta}}\left(s_{\bar{\theta}}+1\right)-3\right] \\
& +\frac{1}{6} V_{8} C_{1}\left[4 s_{\bar{\theta}}\left(s_{\bar{\theta}}+1\right)-3\right]
\end{aligned}
$$

where

$$
C_{1}= \begin{cases}+2 & q_{1}, q_{2}, q \in\{u, d\} \\ -4 & q_{1}, q_{2} \in\{u, d\} \cup q \in s .\end{cases}
$$

Because we consider only $s_{\bar{\theta}}=s_{B}=\frac{1}{2}$ (since $s_{\delta^{\prime}}=0$ in the dynamical diquark model), one can see that both the triquark internal spin-spin coupling $\left(\Delta H_{\kappa_{\delta^{\prime} \bar{Q}}}\right)$ and the $\eta$-like coupling $\left(\Delta H_{V_{8}}\right.$, first introduced in Ref. [36]), are zero. Only the common mass coefficient $M_{0}(1 S)$ and the diquark $\delta$ internal spin coupling $\kappa_{q Q}$ survive. In the order of the $s_{Q \bar{Q}}$ eigenstates $\tilde{P}_{\frac{1}{2}}, \tilde{P}_{\frac{1}{2}}^{\prime}, P_{\frac{3}{2}}$ of Eqs. (6), one finds

$$
\begin{aligned}
& \tilde{M}_{\frac{1-}{2}}=M_{0}\left(\begin{array}{ll}
1 & 0 \\
0 & 1
\end{array}\right)+\frac{1}{2} \kappa_{q Q}\left(\begin{array}{cc}
0 & \sqrt{3} \\
\sqrt{3} & -2
\end{array}\right), \\
& M_{\frac{3}{2}-}=M_{0}+\frac{1}{2} \kappa_{q Q} .
\end{aligned}
$$

The diagonalized form of $M_{\frac{1}{2}}$, which also corresponds to the basis of good $s_{\delta}$ eigenvalues in the order given by Eqs. (3) $\left(P_{\frac{1}{2}}, P_{\frac{1}{2}}^{\prime}, P_{\frac{3}{2}}\right)$, reads

\footnotetext{
${ }^{3}$ Models allowing for all such SU(3) $)_{\text {flavor }}$ exchanges do appear in the literature $[41,45]$.
}

$$
\begin{aligned}
& M_{\frac{1-}{2}}=M_{0}\left(\begin{array}{ll}
1 & 0 \\
0 & 1
\end{array}\right)+\frac{1}{2} \kappa_{q Q}\left(\begin{array}{cc}
-3 & 0 \\
0 & 1
\end{array}\right), \\
& M_{\frac{3}{2}-}=M_{0}+\frac{1}{2} \kappa_{q Q} .
\end{aligned}
$$

One finds (assuming $\kappa_{q Q}>0$ ) a unique $J^{P}=\frac{1}{2}^{-}$ground state and a degenerate pair with $J^{P}=\frac{1}{2}^{-}$and $\frac{3}{2}^{-}$.

\section{B. $P$-wave Hamiltonian and mass expressions}

The $P$-wave states are obtained by combining those in the bases of Eqs. (3) or (6) with a unit $L=1$ of orbital angular momentum. Using the latter basis (unique $s_{Q \bar{Q}}$ eigenvalues), one obtains the seven states in the multiplet $\Sigma^{+}(1 P)$ :

$$
\begin{array}{rlrl}
J^{P} & =\frac{1}{2}^{+}: \tilde{P}_{\frac{1}{2}}^{(L=1)}, & \tilde{P}_{\frac{1}{2}}^{\prime(L=1)}, & P_{\frac{3}{2}}^{(L=1)}, \\
J^{P}=\frac{3}{2}^{+}: \tilde{P}_{\frac{1}{2}}^{(L=1)}, & \tilde{P}_{\frac{1}{2}}^{(L=1)}, & P_{\frac{3}{2}}^{(L=1)}, \\
J^{P}=\frac{5}{2}^{+}: P_{\frac{3}{2}}^{(L=1)} .
\end{array}
$$

Table II collects the quantum numbers of these states, including the total spin $s_{B}$ carried by the baryonic combination $B$ of light quarks $\left(q q_{1} q_{2}\right)$ [which always equals $\frac{1}{2}$ since $\delta^{\prime} \equiv\left(q_{1} q_{2}\right)$ has $s_{\delta^{\prime}}=0$ ], and the total spin $S$ carried

TABLE II. The seven pentaquark $Q \bar{Q} q q_{1} q_{2}$ states in the $\Sigma^{+}(1 P)$ multiplet of the dynamical diquark model, expressed in the basis of good heavy-quark spin $s_{Q \bar{Q}}$. The model restriction that the component diquark $\delta^{\prime} \equiv\left(q_{1} q_{2}\right)$ carries zero spin leads to the baryonic combination $B=\left(q q_{1} q_{2}\right)$ of the light quarks carrying $s_{B}=\frac{1}{2}$ for all states. $S$ is the total spin carried by quarks (the same as the state subscript). Also tabulated are the allowed values of light degree-of-freedom total angular momentum $J_{B}$ and their amplitude contribution $\mathcal{M}_{J_{B}}$ to the full state.

\begin{tabular}{lcccccc}
\hline \hline State & $J^{P}$ & $s_{B}$ & $s_{Q \bar{Q}}$ & $S$ & $J_{B}$ & $\mathcal{M}_{J_{B}}$ \\
\hline$\tilde{P}_{\frac{1}{2}}^{(L=1)}$ & $\frac{1}{2}^{+}$ & $\frac{1}{2}$ & 0 & $\frac{1}{2}$ & $\frac{1}{2}$ & +1 \\
$\tilde{P}_{\frac{1}{2}}^{\prime(L=1)}$ & $\frac{1}{2}^{+}$ & $\frac{1}{2}$ & 1 & $\frac{1}{2}$ & $\frac{1}{2}$ & $-\frac{1}{3}$ \\
$P_{\frac{3}{2}}^{(L=1)}$ & $\frac{1}{2}^{+}$ & $\frac{1}{2}$ & 1 & $\frac{3}{2}$ & $\frac{1}{2}$ & $+\frac{2 \sqrt{2}}{3}$ \\
$\tilde{P}_{\frac{1}{2}}^{(L=1)}$ & & & & & $\frac{3}{2}$ & $+\frac{1}{3}$ \\
$\tilde{P}_{\frac{1}{2}}^{\prime(L=1)}$ & $\frac{3}{2}+$ & $\frac{1}{2}$ & 0 & $\frac{1}{2}$ & $\frac{3}{2}$ & +1 \\
$P_{\frac{3}{2}}^{(L=1)}$ & & 1 & $\frac{1}{2}$ & $\frac{1}{2}$ & $-\frac{2}{3}$ \\
$P^{(L=1)}$ & & $\frac{1}{2}+$ & 1 & $\frac{3}{2}$ & $\frac{1}{2}$ & $+\frac{\sqrt{5}}{3}$ \\
\hline \hline
\end{tabular}


by all quarks. In addition, we tabulate for completeness the relative amplitude $\mathcal{M}_{J_{B}}$ within each state for each allowed eigenvalue $J_{B}$ of total angular momentum $\boldsymbol{J}_{B} \equiv \boldsymbol{L}+\boldsymbol{s}_{B}$ carried by the light degrees of freedom. These recoupling amplitudes are given by

$$
\begin{aligned}
\mathcal{M}_{J_{B}} & \equiv\left\langle\left(L, s_{B}\right), J_{B}, s_{Q \bar{Q}}, J \mid L,\left(s_{B}, s_{Q \bar{Q}}\right), S, J\right\rangle \\
& =(-1)^{L+s_{B}+s_{Q \bar{Q}}+J} \sqrt{\left[J_{B}\right][S]}\left\{\begin{array}{lll}
L & s_{B} & J_{B} \\
s_{Q \bar{Q}} & J & S
\end{array}\right\} .
\end{aligned}
$$

While a decomposition in $J_{B}$ is not needed for the following mass analysis, we anticipate its potential usefulness for computing transition matrix elements, as is done for the tetraquark states in Ref. [44].

The minimal Hamiltonian for the first excited pentaquark multiplet, $\Sigma^{+}(1 P)$, closely follows the one used for the tetraquark multiplet $\Sigma_{g}^{+}(1 P)$ in Ref. [33]:

$$
\begin{aligned}
H= & M_{0}+\Delta H_{\kappa_{q Q}}+\Delta H_{\kappa_{\delta^{\prime}}}+\Delta H_{V_{L S}}+\Delta H_{V_{T}}+\Delta H_{V_{8}} \\
= & M_{0}+2\left[\kappa_{q Q}\left(\vec{s}_{q} \cdot \vec{s}_{Q}\right)+\kappa_{\delta^{\prime}}\left(\vec{s}_{\delta^{\prime}} \cdot \vec{s}_{\bar{Q}}\right)\right] \\
& +V_{L S} \vec{L} \cdot \vec{S}+V_{T} \hat{S}_{12}^{(\delta \bar{\theta})}+V_{8}\left(\lambda_{a}^{8} \vec{\sigma}_{\delta^{\prime}}\right) \cdot\left(\lambda_{a}^{8} \vec{\sigma}_{q}\right) .
\end{aligned}
$$

In addition to a common mass $\left[M_{0}(1 P)\right.$ here], we allow for internal spin-spin couplings for the diquark $\delta\left(\kappa_{q Q}\right)$ and the triquark $\bar{\theta}\left(\kappa_{\delta^{\prime}}\right)$, as well as a spin-orbit term $V_{L S}$, a tensor term $V_{T}$, and an $\eta$-like exchange term labeled by $V_{8}$ [36]. In addition, the numerical value of $\kappa_{q Q}$ in the $1 P$ multiplet is expected to differ from that in the $1 S$ multiplet [33]. The matrix elements of the mass Hamiltonian read:

$$
\begin{aligned}
M= & M_{0}+\frac{\kappa_{q Q}}{2}\left[2 s_{\delta}\left(s_{\delta}+1\right)-3\right]+\frac{\kappa_{\delta^{\prime}} \bar{Q}}{4}\left[4 s_{\bar{\theta}}\left(s_{\bar{\theta}}+1\right)-3\right] \\
& +\frac{V_{L S}}{2}[J(J+1)-L(L+1)-S(S+1)] \\
& +V_{T}\left\langle S_{12}^{(\delta \bar{\theta})}\right\rangle+\frac{1}{6} V_{8} C_{1}\left[4 s_{B}\left(s_{B}+1\right)-3\right] .
\end{aligned}
$$

The tensor term labeled by $V_{T}$ differs somewhat from the primary one studied in Ref. [33], for which the spins entering the operator are those of the individual light quarks $q, \bar{q}$ within the diquarks. Instead, the tensor operator used here couples directly to the full quasiparticle $\delta, \bar{\theta}$ spins, and thus is the analog of the secondary possible tensor operator studied in Ref. [33], Appendix A. Its matrix elements are computed as

$$
\begin{aligned}
\left\langle L^{\prime}, S^{\prime}, J\left|\hat{S}_{12}^{(\delta \bar{\theta})}\right| L, S, J\right\rangle= & (-1)^{S+J} \sqrt{30[L]\left[L^{\prime}\right][S]\left[S^{\prime}\right]}\left\{\begin{array}{ccc}
J & S^{\prime} & L^{\prime} \\
2 & L & S
\end{array}\right\}\left(\begin{array}{ccc}
L^{\prime} & 2 & L \\
0 & 0 & 0
\end{array}\right)\left\{\begin{array}{ccc}
s_{\bar{\theta}} & s_{\delta} & S \\
s_{\bar{\theta}}^{\prime} & s_{\delta}^{\prime} & S^{\prime} \\
1 & 1 & 2
\end{array}\right\} \\
& \times\left\langle s_{\bar{\theta}}^{\prime}\left\|\boldsymbol{\sigma}_{\bar{\theta}}\right\| s_{\bar{\theta}}\right\rangle\left\langle s_{\delta}^{\prime}\left\|\boldsymbol{\sigma}_{\delta}\right\| s_{\delta}\right\rangle .
\end{aligned}
$$

Here, $\sigma$ denotes not just spin- $\frac{1}{2}$ Pauli matrices, but more generally twice the canonically normalized generators $\mathbf{s}$ for arbitrary spin $s$, and the reduced matrix elements of the angular momentum generators are given by

$$
\left\langle j^{\prime}|| \mathbf{j}|| j\right\rangle=\sqrt{j(2 j+1)(j+1)} \delta_{j^{\prime} j} .
$$

In particular, if $s_{\delta}=0$, then the reduced matrix element (and hence $\left\langle\hat{S}_{12}^{(\delta \bar{\theta})}\right\rangle$ ) vanishes.

Just as in the case of the $S$-wave states, one sees that both $\Delta H_{\kappa_{\delta^{\prime} \bar{Q}}}=\Delta H_{V_{8}}=0$, and the surviving matrix elements of Eq. (14) for the $\Sigma^{+}(1 P)$ multiplet, expressed in the same order as the states listed in Eq. (12) or Table II, then read

$$
\begin{aligned}
& \tilde{M}_{\frac{1}{2}}=M_{0}\left(\begin{array}{lll}
1 & 0 & 0 \\
0 & 1 & 0 \\
0 & 0 & 1
\end{array}\right)+\frac{\kappa_{q Q}}{2}\left(\begin{array}{ccc}
-2 & \sqrt{3} & 0 \\
\sqrt{3} & 0 & 0 \\
0 & 0 & 1
\end{array}\right)-\frac{V_{L S}}{2}\left(\begin{array}{ccc}
2 & 0 & 0 \\
0 & 2 & 0 \\
0 & 0 & 5
\end{array}\right)+V_{T}\left(\begin{array}{ccc}
0 & 0 & \sqrt{6} \\
0 & 0 & \sqrt{2} \\
\sqrt{6} & \sqrt{2} & -4
\end{array}\right), \\
& \tilde{M}_{\frac{3}{2}+}=M_{0}\left(\begin{array}{lll}
1 & 0 & 0 \\
0 & 1 & 0 \\
0 & 0 & 1
\end{array}\right)+\frac{\kappa_{q Q}}{2}\left(\begin{array}{ccc}
-2 & \sqrt{3} & 0 \\
\sqrt{3} & 0 & 0 \\
0 & 0 & 1
\end{array}\right)+\frac{V_{L S}}{2}\left(\begin{array}{ccc}
1 & 0 & 0 \\
0 & 1 & 0 \\
0 & 0 & -2
\end{array}\right)+\frac{V_{T}}{5}\left(\begin{array}{ccc}
0 & 0 & -\sqrt{15} \\
0 & 0 & -\sqrt{5} \\
-\sqrt{15} & -\sqrt{5} & 16
\end{array}\right), \\
& M_{\frac{5}{2}+}=M_{0}+\frac{1}{2} \kappa_{q Q}+\frac{3}{2} V_{L S}-\frac{4}{5} V_{T} .
\end{aligned}
$$

These matrices, once diagonalized, provide the mass eigenvalues: 


$$
\begin{aligned}
& M_{\frac{1}{2}+}=M_{0}\left(\begin{array}{ccc}
1 & 0 & 0 \\
0 & 1 & 0 \\
0 & 0 & 1
\end{array}\right)+\frac{\kappa_{q Q}}{2}\left(\begin{array}{ccc}
1 & 0 & 0 \\
0 & -3 & 0 \\
0 & 0 & 1
\end{array}\right)-\frac{1}{4} V_{L S}\left(\begin{array}{ccc}
7 & 0 & 0 \\
0 & 4 & 0 \\
0 & 0 & 7
\end{array}\right)-V_{T}\left(\begin{array}{ccc}
2 & 0 & 0 \\
0 & 0 & 0 \\
0 & 0 & 2
\end{array}\right)+\frac{\sqrt{3}}{4} \tilde{V}_{1}\left(\begin{array}{ccc}
-1 & 0 & 0 \\
0 & 0 & 0 \\
0 & 0 & 1
\end{array}\right), \\
& M_{\frac{3}{2}+}=M_{0}\left(\begin{array}{ccc}
1 & 0 & 0 \\
0 & 1 & 0 \\
0 & 0 & 1
\end{array}\right)+\frac{\kappa_{q Q}}{2}\left(\begin{array}{ccc}
1 & 0 & 0 \\
0 & -3 & 0 \\
0 & 0 & 1
\end{array}\right)-\frac{1}{4} V_{L S}\left(\begin{array}{ccc}
1 & 0 & 0 \\
0 & -2 & 0 \\
0 & 0 & 1
\end{array}\right)+\frac{8}{5} V_{T}\left(\begin{array}{ccc}
1 & 0 & 0 \\
0 & 0 & 0 \\
0 & 0 & 1
\end{array}\right)+\frac{\sqrt{3}}{20} \tilde{V}_{2}\left(\begin{array}{ccc}
-1 & 0 & 0 \\
0 & 0 & 0 \\
0 & 0 & 1
\end{array}\right) \text {, } \\
& M_{\frac{5}{2}}=M_{0}+\frac{1}{2} \kappa_{q Q}+\frac{3}{2} V_{L S}-\frac{4}{5} V_{T} \text {, }
\end{aligned}
$$

where

$$
\begin{gathered}
\tilde{V}_{1}=\sqrt{3 V_{L S}^{2}+16 V_{L S} V_{T}+64 V_{T}^{2}}, \\
\tilde{V}_{2}=\sqrt{75 V_{L S}^{2}-320 V_{L S} V_{T}+448 V_{T}^{2}} .
\end{gathered}
$$

The elements of these diagonalized matrices are presented in the order of increasing mass eigenvalues in each $J^{P}$ sector, under the assumption (as found for tetraquarks in Ref. [33]) that the contribution from $V_{L S}$ dominates the contribution from $\kappa_{q Q}$ (and from $V_{T}$ ).

Anticipating results from the analysis in the next section, we also present the corresponding expressions to Eq. (19) when $V_{T} \rightarrow 0$. Including up to linear order in $V_{T}$, one finds

$$
\begin{aligned}
& M_{\frac{1}{2}}=M_{0}\left(\begin{array}{lll}
1 & 0 & 0 \\
0 & 1 & 0 \\
0 & 0 & 1
\end{array}\right)+\frac{\kappa_{q Q}}{2}\left(\begin{array}{ccc}
1 & 0 & 0 \\
0 & -3 & 0 \\
0 & 0 & 1
\end{array}\right)-\frac{1}{2} V_{L S}\left(\begin{array}{ccc}
5 & 0 & 0 \\
0 & 2 & 0 \\
0 & 0 & 2
\end{array}\right)-4 V_{T}\left(\begin{array}{lll}
1 & 0 & 0 \\
0 & 0 & 0 \\
0 & 0 & 0
\end{array}\right), \\
& M_{\frac{3}{2}+}=M_{0}\left(\begin{array}{lll}
1 & 0 & 0 \\
0 & 1 & 0 \\
0 & 0 & 1
\end{array}\right)+\frac{\kappa_{q Q}}{2}\left(\begin{array}{ccc}
1 & 0 & 0 \\
0 & -3 & 0 \\
0 & 0 & 1
\end{array}\right)+\frac{1}{2} V_{L S}\left(\begin{array}{ccc}
-2 & 0 & 0 \\
0 & 1 & 0 \\
0 & 0 & 1
\end{array}\right)+\frac{16}{5} V_{T}\left(\begin{array}{lll}
1 & 0 & 0 \\
0 & 0 & 0 \\
0 & 0 & 0
\end{array}\right), \\
& M_{\frac{5}{2}+}=M_{0}+\frac{1}{2} \kappa_{q Q}+\frac{3}{2} V_{L S}-\frac{4}{5} V_{T} .
\end{aligned}
$$

It is worth emphasizing that the Hamiltonian of Eq. (14) with $V_{T}=0$ is diagonal in the basis of good $s_{\delta}$ defined in Eqs. (3) [as is apparent from Eq. (15), since the $\kappa_{\delta^{\prime} \bar{Q}}$ and $V_{8}$ terms are also absent when $\left.s_{\delta^{\prime}}=0\right]$. The mass expressions of Eqs. (21) with $V_{T}=0$ therefore refer to the diquark-spin basis of Eqs. (3), specifically, in the order $P_{\frac{3}{2}}, P_{\frac{1}{2}}, P_{\frac{1}{2}}^{\prime}$.

\section{ANALYSIS}

Using the results of the previous section, particularly Eqs. (11), (19), and (20), we compute the mass eigenvalues for all pentaquarks with flavor content $c \bar{c} q q_{1} q_{2}$ and $c \bar{c} u d s$ in the ground-state multiplet $\left[\Sigma^{+}(1 S)\right]$ given in Eqs. (3) or (6), and the first excited-state multiplet $\left[\Sigma^{+}(1 P)\right]$ given in Eqs. (12). The measured masses of the four narrow states $\left[P_{c}(4312), P_{c}(4337), P_{c}(4440)\right.$, and $\left.P_{c}(4457)\right]$ in Table I are used to assign these states to the multiplet $\Sigma^{+}(1 P)$, while we defer a discussion of the problematic wide $P_{c}(4380)$ until later in this section.
The assignment of the known $P_{c}$ states to $\Sigma^{+}(1 P)$ rather than $\Sigma^{+}(1 S)$ in the dynamical diquark model was first noted in Ref. [31] to be much more natural, despite the lack of clear experimental evidence for the lighter $S$-wave states. In that work, the argument rested upon the opposite-parity nature of the (unresolved) $P_{c}(4450)$ and $P_{c}(4380)$. Now, the $\Sigma^{+}(1 P)$ assignment can be made based upon the sheer multiplicity of $P_{c}$ states: $\Sigma^{+}(1 S)$ has only three states [Eq. (3)], so that at least some of the $P_{c}$ 's must belong to a higher multiplet. However, the model predicts a $1 P-1 S$ mass splitting of $\sim 400 \mathrm{MeV}$, rendering a mixed $1 P-1 S$ assignment of the known $P_{c}$ states untenable. In fact, the closely (and almost equally) spaced pairs $P_{c}$ (4312)- $P_{c}(4337)$ and $P_{c}(4440)-P_{c}(4457)$ have a completely natural identification within $\Sigma^{+}(1 P)$ : Anticipating our result that $V_{T}$ turns out to be numerically small, and using the $V_{T} \rightarrow 0$ expressions of Eq. (21), one sees that the heaviest $\frac{1}{2}^{+}$and lightest $\frac{3}{2}^{+}$states are nearly degenerate: 


$$
m_{\frac{3}{2}+}^{(1)}-m_{\frac{1}{2}+}^{(3)}=\frac{36}{5} V_{T},
$$

and that, for $V_{T}=0$, one finds two equally spaced pairs:

$$
m_{\frac{1+}{2}}^{(2)}-m_{\frac{1+}{2}}^{(1)}=m_{\frac{3+}{2}+}^{(2)}-m_{\frac{3+}{2}}^{(1)}=\frac{3}{2} V_{L S}-2 \kappa_{q Q}
$$

In contrast, the other three mass splittings of consecutive states in $\Sigma^{+}(1 P)$ equal $V_{L S}$ or $\frac{3}{2} V_{L S}$. The analysis of hidden-charm $P$-wave tetraquarks in Ref. [33] produces values $V_{L S} \simeq 45-60 \mathrm{MeV}$ and $\kappa_{q c}(1 P)=40-45 \mathrm{MeV}$, so that typically, $\left|\frac{3}{2} V_{L S}-2 \kappa_{q c}\right| \ll V_{L S}$. Assuming that the corresponding hidden-charm nonstrange $P$-wave pentaquarks produce similar numerical values for the coefficients $V_{L S}$ and $\kappa_{q c}(1 P)$ (as they must, since they arise from the same dynamics), one expects the seven masses in the $\Sigma^{+}(1 P)$ spectrum to appear as two nearly degenerate values [Eq. (22)], two closely spaced pairs [Eq. (23)], and two heavier masses, $m_{\frac{3+}{2}}^{(3)}$ and $m_{\frac{5}{2}+}$.

\section{A. The $c \bar{c} q q_{1} q_{2}$ sector, $P$ wave}

Having thus identified specific candidates for the four known $P_{c}$ states:

$$
\begin{aligned}
& P_{c}(4312)=P_{\frac{1}{2}}^{(1)}, \\
& P_{c}(4337)=P_{\frac{1}{2}}^{(2)}, \\
& P_{c}(4440)=P_{\frac{3}{2}}^{(1)} \text { and } P_{\frac{1}{2}}^{(3)}, \\
& P_{c}(4457)=P_{\frac{3}{2}}^{(2)},
\end{aligned}
$$

we first perform a least-squares fit to the $V_{T}=0$ mass expressions of Eqs. (21) and obtain values for $M_{0}(1 P)$, $V_{L S}$, and $\kappa_{q c}(1 P)$. We then predict the masses for the remaining states of $\Sigma^{+}(1 P)$. The results are presented in Table III.

Since the $V_{T}=0$ fit produces a figure of merit $\chi_{\min }^{2}<1$, we conclude that the parameter $V_{T}$ is not actually needed for a complete description of the currently available $P_{c}$ data. Nevertheless, we perform a fit to the full expressions of Eqs. (19)-(20), which include $V_{T}$ nonlinearly. Since four parameters are fit by four masses in that case, the solution is unique, and we therefore do not propagate the uncertainties on the parameters or masses for that fit. These results are also presented in Table III. We find the essential result that $V_{T}$ is not merely statistically unimportant (as shown by the $V_{T}=0$ fit), but also that its value in a fit with no free parameters is numerically small compared to that of the other Hamiltonian parameters $\left[M_{0}, V_{L S}, \kappa_{q c}(1 P)\right]$. The most incisive test for the existence of a nonzero $V_{T}$, as indicated by Eqs. (22) and (24), would be the resolution
TABLE III. Calculations of Hamiltonian parameters and masses for the $c \bar{c} q q_{1} q_{2} \Sigma^{+}(1 P)$ states. All masses are in units of $\mathrm{MeV}$. The fit in the first column sets the tensor coupling $V_{T}=0$. Boldface indicates best-fit masses to measured values from Table I.

\begin{tabular}{lcc}
\hline \hline$\chi_{\min }^{2}$ & 0.468 & 0.000 \\
$M_{0}(1 P)$ & $4495.4 \pm 6.4$ & 4492.0 \\
$m_{\delta=(c q)}$ & $1927.1 \pm 11.0$ & $1927.1 \pm 11.0$ \\
$m_{\bar{\theta}}$ & $2077.2 \pm 11.6$ & $2073.5 \pm 9.0$ \\
$\kappa_{q c}(1 P)$ & $52.4 \pm 6.3$ & 49.9 \\
$V_{L S}$ & $82.8 \pm 5.6$ & 80.2 \\
$V_{T}$ & 0.0 & 1.1 \\
$M_{\frac{1}{2}+}$ & $\mathbf{4 3 1 4 . 6} \pm 6.8$ & $\mathbf{4 3 1 1 . 9}$ \\
& $\mathbf{4 3 3 4 . 1} \pm 9.4$ & $\mathbf{4 3 3 7 . 0}$ \\
$M_{\frac{3}{2}+}$ & $4438.8 \pm 4.9$ & 4436.8 \\
& $\mathbf{4 4 3 8 . 8} \pm 4.9$ & $\mathbf{4 4 4 0 . 3}$ \\
& $\mathbf{4 4 5 8 . 2} \pm 4.1$ & $\mathbf{4 4 5 7 . 3}$ \\
$M_{\frac{5}{2}}$ & $4563.0 \pm 11.9$ & 4557.3 \\
\hline \hline
\end{tabular}

of $P_{c}(4440)$ into a very closely spaced $\frac{1}{2}+, \frac{3}{2}+$ pair. Indeed, the difference between the $\frac{3}{2}+$ and $\frac{1}{2}+$ sides of Eq. (23), using Eqs. (19)-(20), is $O\left(V_{T}^{2} / V_{L S}\right)$; in comparison, its experimental value using the masses in Table I is $-8.1 \pm 11.9 \mathrm{MeV}$.

The hidden-charm $P$-wave pentaquarks studied here share several similarities with the hidden-charm $P$-wave tetraquarks studied in Ref. [33] within the dynamical diquark model, but also feature some significant differences. Both are modeled as heavy (and therefore effectively semistatic) color-triplet quasiparticles connected by the same orbitally excited $(L=1)$ color flux tube, whose excitation energies are computed as BO potentials obtained from specific lattice simulations, labeled here as JKM (Refs. [46,47]) and CPRRW (Ref. [48]). In both pentaquark and tetraquark cases, the known states occupy the $1 P$ levels of the ground-state $\mathrm{BO}$ potential $\Sigma^{+}$, but since each tetraquark consists of a $[\delta=(c q)]-[\bar{\delta}=(\bar{c} \bar{q})]$ pair, the tetraquark $\mathrm{BO}$ potential is labeled by an additional $C P$ eigenvalue: $\Sigma_{g}^{+}$. In addition, the nonstrange pentaquark states in this analysis all have $I=\frac{1}{2}$ because the diquark $\delta^{\prime}$ internal to the triquark $\bar{\theta}$ is assumed to be isoscalar, so that the overall isospin of the state is carried by the light quark in $\delta$. The tetraquarks, in contrast, have isospin dependence via interactions between the light quarks in the $\delta-\bar{\delta}$ pair (via both spin-spin and tensor terms).

The two systems also feature some of the same operators in their Hamiltonians, specifically the internal diquark spin coupling $\kappa_{q c}(1 P)$ and the spin-orbit coupling $V_{L S}$. The analysis of the hidden-charm $P$-wave tetraquarks is challenging because the 4 predicted $1^{--}$states in $\Sigma_{g}^{+}(1 P)$ can be assigned to observed states [e.g., $Y(4220)$ ] in a variety of 
ways [33], while as noted above, the analysis of the hiddencharm $P$-wave pentaquarks is challenging due to a lack of measured $J^{P}$ quantum numbers for any of the states. Nevertheless, the numerical values of $V_{L S}$ and $\kappa_{q c}(1 P)$ for the two cases are quite comparable: $V_{L S} \simeq 45-60 \mathrm{MeV}$ and $\kappa_{q c}(1 P)=40-45 \mathrm{MeV}$ for the most plausible fits in Ref. [33], as compared with $V_{L S} \simeq 80 \mathrm{MeV}$ and $\kappa_{q c}(1 P) \simeq$ $50 \mathrm{MeV}$ from Table III.

Predictions for the masses of all seven states in the $\Sigma^{+}(1 P)$ multiplet are presented in Table III. $P_{c}(4312)$ is seen to be the lightest state of the multiplet, $P_{c}(4337)$ is the next lightest, followed by the nearly degenerate pair coinciding with $P_{c}(4440)$, and then $P_{c}(4457)$. Notably, no lower or intermediate states appear. Finally, the heaviest $\frac{3}{2}+$ and the $\frac{5}{2}^{+}$lie much higher in mass; LHCb does present $J / \psi p$ data from $\Lambda_{b}$ decays up to almost $5 \mathrm{GeV}$ [13], but the statistics appear insufficient to resolve states beyond about $4500 \mathrm{MeV}$.

Notably, no signal for $P_{c}(4337)$ is apparent in the LHCb $\Lambda_{b}$ decay data, but only in their $B_{s}^{0}$ decay data [14]. On the other hand, Ref. [14] sees no signal for $P_{c}(4312)$, and limited phase space precludes observation of the higher $P_{c}$ states. These curious results from the same facility, taken at face value, suggest different internal wave-function structures for $P_{c}(4312)$ and $P_{c}(4337)$ being accessed through different processes. Recalling that the $V_{T}=0$ mass eigenstates are those of good diquark spin $s_{\delta}$ [Eq. (3)], the $\mathrm{LHCb}$ data can be explained if $\Lambda_{b}$ decays preferentially couple to states with $s_{\delta}=1$ [and hence to $P_{\frac{3}{2}}$, the core quark state of $P_{c}(4312)$ ], while $B_{s}^{0}$ decays preferentially couple to states with $s_{\delta}=0$ [and hence to $P_{\frac{1}{2}}$, the core quark state of $\left.P_{c}(4337)\right]$. While the dynamical explanations for these couplings are not immediately clear, one may observe that the decay $B_{s}^{0} \rightarrow J / \psi p \bar{p}$ requires the annihilation of the initial valence quarks $\bar{b} s \rightarrow \bar{c} c$ through the $t$-channel exchange of a single virtual $W$ boson, while the initial $\Lambda_{b}$ light valence quarks $u d$ in the dynamical diquark model persist through the decay as the "good" diquark $\delta^{\prime}$. Such differences could certainly have a pronounced effect upon the internal spin structure of the produced states.

Information can also be obtained from the quarkonium decays of the states, assuming the conservation of heavyquark spin $s_{Q \bar{Q}}$. The underlying quark states $P_{\frac{1}{2}}, P_{\frac{1}{1}}^{\prime}, P_{\frac{3}{2}}$ of Eqs. (3), which we have found to coincide with the mass eigenstates in the limit $V_{T}=0$, are decomposed in terms of $s_{Q \bar{Q}}$ eigenvalues in Eqs. (5). Thus, for example, $P_{c}(4457)$ coincides with $P_{\frac{3}{2}}$, which has only an $s_{Q \bar{Q}}=1$ component. Therefore, $P_{c}$ (4457) should (and does) decay prominently to $J / \psi p$, but not to $\eta_{c} p$.

Lastly, we consider the troublesome $P_{c}(4380)$ opposite-parity signal. With the narrow $P_{c}$ states filling the positive-parity $\Sigma^{+}(1 P)$ multiplet, the very broad $P_{c}(4380)$ presumably belongs to a negative-parity $S$-wave multiplet.
However, calculations of multiplet-average masses in the dynamical diquark model [31] predict the $\Sigma^{+}(1 S)$ masses to be nearly $400 \mathrm{MeV}$ lower and the $\Sigma^{+}(2 S)$ masses to be nearly $200 \mathrm{MeV}$ higher than those in $\Sigma^{+}(1 P)$. We confirm these results in this latest analysis, incorporating the best determination of the $(c q)$ diquark mass from the latest analysis of $c \bar{c} q^{\prime} \bar{q}$ tetraquarks in Ref. [36],

$$
\begin{array}{rll}
m_{\delta(c q)} & =1938.0 \pm 0.9 \mathrm{MeV} & (\mathrm{JKM}), \\
& =1916.2 \pm 0.9 \mathrm{MeV} \quad(\mathrm{CPRRW}),
\end{array}
$$

to obtain the triquark masses $m_{\bar{\theta}}$ presented in Table III. If $P_{c}(4380)$ survives further analysis as a distinct state, it cannot be of the same diquark-triquark structure as the other $P_{c}$ states; the most likely candidate would then be a $\bar{D} \Sigma_{c}^{*}$ threshold effect or molecule [51], which in its relative $S$ wave has the required negative parity.

\section{B. The $c \bar{c} q q_{1} q_{2}$ sector, $S$ wave}

With specific diquark masses $m_{\delta}$ [Eqs. (25)] and triquark masses $m_{\bar{\theta}}$ (Table III) in hand, one can solve the coupled Schrödinger equations in the dynamical diquark model for all $\mathrm{BO}$ potentials, as is done in Ref. [31]. Here, we extract the value of $M_{0}(1 S)$, which is one of the two parameters appearing in the mass expressions of Eqs. (11), and present it in Table IV. The only additional parameter then needed to predict all state masses in the $\Sigma^{+}(1 S)$ multiplet is $\kappa_{q c}(1 S)$, which can be obtained from the latest hidden-charm tetraquark analysis of Ref. [36]:

$$
\kappa_{q c}(1 S)=25.6 \pm 5.0 \mathrm{MeV} .
$$

The masses of the three states of $\Sigma^{+}(1 S)$ obtained from these values are presented in Table IV.

Of course, no hidden-charm pentaquark states with such low masses have yet been observed. However, one notes two important points in this regard: First, the region below about $4100 \mathrm{MeV}$ in the data of Ref. [13] has rapidly vanishing $J / \psi p$ phase space (threshold at $4035 \mathrm{MeV}$ ), meaning that the ground state at $\simeq 4085 \mathrm{MeV}$ might be difficult to discern. Even so, the LHCb data from about 4100-4200 MeV appears as a broad enhancement that could easily contain signals of resonances. The $\Sigma^{+}(1 S)$ states, carrying negative parity, are not protected by a centrifugal barrier against $S$-wave fall-apart decays into $J / \psi p$, and therefore can have substantial widths. Second, the proposed suppression of $\Lambda_{b}$ decays to $\Sigma^{+}(1 P)$ states with $s_{\delta}=0$, if it holds for the multiplet $\Sigma^{+}(1 S)$, eliminates the ground state $P_{\frac{1}{2}}$ in Eqs. (3) from appearing in $\Lambda_{b}$ decays,

\footnotetext{
${ }^{4}$ These values are quite comparable to those obtained from other determinations, such as $m_{\delta(c q)}=1975 \mathrm{MeV}$ from a constituent-quark approach [49] or $1860 \pm 50 \mathrm{MeV}$ from QCD sum rules [50].
} 
TABLE IV. Calculations of Hamiltonian parameters and masses for the $c \bar{c} q q_{1} q_{2} \Sigma^{+}(1 S)$ states. All masses are in units of $\mathrm{MeV}$. The fit in the first (second) column uses values in which the corresponding $M_{0}(1 P)$ value (as well as $m_{\delta}, m_{\bar{\theta}}$ ) is obtained from the first (second) column of Table III, respectively.

\begin{tabular}{lcc}
\hline \hline$M_{0}(1 S)$ & $4123.6 \pm 1.7$ & $4127.0 \pm 1.7$ \\
$m_{\delta=(c q)}$ & $1927.1 \pm 11.0$ & $1927.1 \pm 11.0$ \\
$m_{\bar{\theta}}$ & $2077.2 \pm 11.6$ & $2073.5 \pm 9.0$ \\
$\kappa_{q c}(1 S)$ & $25.6 \pm 5.0$ & $25.6 \pm 5.0$ \\
$M_{\frac{1}{2}-}$ & $4085.2 \pm 7.7$ & $4088.6 \pm 7.7$ \\
& $4136.4 \pm 3.0$ & $4139.8 \pm 3.0$ \\
$M_{\frac{3}{2}-}$ & $4136.4 \pm 3.0$ & $4139.8 \pm 3.0$ \\
\hline \hline
\end{tabular}

leaving only the (nearly degenerate) heavier $\frac{1}{2}^{-}-\frac{3}{2}^{-}$pair, $P_{\frac{1}{2}}^{\prime}$ and $P_{\frac{3}{2}}$. The LHCb data from $B_{s}^{0}$ decays [14] has substantially larger statistical uncertainties than that from $\Lambda_{b}$ decays, but even so, hints at some possible structures in the range of 4100-4200 MeV.

\section{The $c \bar{c} u d s$ Sector}

Using the same triquark masses $m_{\bar{\theta}}$ as in Table IV and the (cs) diquark mass $m_{\delta(c s)}$ obtained from the hidden-charm, open-strange tetraquark states $Z_{c s}$ in Ref. [36],

$$
\begin{array}{rll}
m_{\delta(c s)} & =2080.2 \pm 1.5 \mathrm{MeV} & (\mathrm{JKM}), \\
& =2058.5 \pm 1.5 \mathrm{MeV} \quad(\mathrm{CPRRW})
\end{array}
$$

one may immediately compute the $c \bar{c} u d s$ multiplet-average masses $M_{0}(1 P)$ and $M_{0}(1 S)$ for $\Sigma^{+}(1 P)$ and $\Sigma^{+}(1 S)$, respectively, just as done in the previous subsections. We also have the value of $\kappa_{s c}(1 S)$ from Ref. [36],

$$
\kappa_{s c}(1 S)=109.8 \pm 1.1 \mathrm{MeV},
$$

which permits the immediate computation of the three $c \bar{c} u d s$ states in $\Sigma^{+}(1 S)$.

However, we possess no independent determination of $\kappa_{s c}(1 P)$, since no $P$-wave $c \bar{c} q \bar{s}$ candidates have yet been observed. The large value of $\kappa_{s c}(1 S)$ in Eq. (28), as compared to the value of $\kappa_{q c}(1 S)$ in Eq. (26), is argued in Ref. [33] to result from the heavier $s$ quark forming a (cs) diquark that is more compact than $(c q)$, which therefore subjects the $c, s$ quarks to much larger spin-spin couplings. Meanwhile, the larger value of $\kappa_{q c}(1 P)$ $(\simeq 50 \mathrm{MeV}$ from Table III), as compared to that of $\kappa_{q c}(1 S)$ in Eq. (26), has been discussed in Ref. [33] as a possible result of finite diquark sizes leading to a sensitivity of their internal spin couplings on the angular momentum of the flux tube connecting them. One expects this sensitivity to be greater for diquarks containing a light quark $q$ than an $s$ quark. Additionally, the $P$-wave Hamiltonian of Eq. (14) includes the spin-orbit coupling
TABLE V. Calculations of Hamiltonian parameters and masses for the $c \bar{c} s q_{1} q_{2} \Sigma^{+}(1 P)$ states. All masses are in units of MeV. The fit in the first column sets the tensor coupling $V_{T}=0$. The triquark mass $m_{\bar{\theta}}$ values are obtained from the corresponding column of Table III.

\begin{tabular}{lcc}
\hline \hline$M_{0}(1 P)$ & $4624.7 \pm 0.0$ & $4621.3 \pm 0.0$ \\
$m_{\delta=(c s)}$ & $2069.4 \pm 10.9$ & $2069.4 \pm 10.9$ \\
$m_{\bar{\theta}}$ & $2077.2 \pm 11.6$ & $2073.5 \pm 9.0$ \\
$\kappa_{q c}(1 P)$ & $52.4 \pm 6.3$ & 49.9 \\
$V_{L S}$ & $82.8 \pm 5.6$ & 80.2 \\
$V_{T}$ & 0.0 & 1.1 \\
$M_{\frac{1}{2}+}$ & $4444.0 \pm 14.4$ & 4441.3 \\
& $4463.4 \pm 11.0$ & 4466.3 \\
$M_{\frac{3}{2}+}$ & $4568.1 \pm 6.4$ & 4566.2 \\
& $4568.1 \pm 6.4$ & 4569.6 \\
& $4587.6 \pm 9.9$ & 4586.6 \\
$M_{\frac{5}{2}+}$ & $4692.3 \pm 4.2$ & 4686.4 \\
\hline \hline
\end{tabular}

$V_{L S}$, whose value might very well depend upon whether $(c s)$ or $(c q)$ diquarks are present. For the analysis of the $\Sigma^{+}(1 P) c \bar{c} u d s$ states, we simply adopt the numerical values of the corresponding Hamiltonian couplings from the $\Sigma^{+}(1 P) c \bar{c} q q_{1} q_{2}$ system given in Table III. The results are presented in Table V.

One notes immediately from Table $\mathrm{V}$ that the mass predictions for the lightest two $\frac{1}{2}^{+}$states are separated by only about $20 \mathrm{MeV}$, and they bracket the measured mass of $P_{c s}(4459)$ given in Table I. These states are the strange analogues of $P_{c}(4312)$ and $P_{c}(4337)$. Indeed, since the production of $P_{c s}(4459)$ via $\Xi_{b}^{-} \rightarrow(J / \psi \Lambda) K^{-}$[15] is a strange analogue to the production of $P_{c}(4312)$ via $\Lambda_{b} \rightarrow(J / \psi p) K^{-}$, one might expect that the absence of $P_{c}$ (4337) in the latter predicts the absence of the secondlightest $\frac{1}{2}+P_{c s}$ state in the former. The identification of $P_{c s}(4459)$ as the strange analogue of $P_{c}(4312)$ in the dynamical diquark model was proposed in Ref. [36]. Alternately, since the $J / \psi \Lambda$ data does not yet have the same level of statistics as the $J / \psi p$ data, the possibility of two closely spaced $\frac{1}{2}^{+}$states near $4459 \mathrm{MeV}$ awaiting resolution is a very real possibility. ${ }^{5}$ The other $\Sigma^{+}(1 P) P_{c s}$ states in this calculation are over $100 \mathrm{MeV}$ heavier, and thus far only appear in the LHCb data [15] as tantalizing hints.

The predictions for the $\Sigma^{+}(1 S)$ states, using Eq. (28), are presented in Table VI. Again, the statistics of the LHCb data in Ref. [15] are not yet sufficient to determine whether significant $P_{c s}$ peaks reside in this mass range. Similar comments to those for the $\Sigma^{+}(1 S) P_{c}$ states apply in this case (noting that the $J / \psi \Lambda$ threshold is $4213 \mathrm{MeV}$ ).

\footnotetext{
${ }^{5} \mathrm{~A}$ similar scenario is proposed in the molecular picture [52], except in that case referring to a $\frac{1}{2}^{-}-\frac{3}{2}^{-}$pair.
} 
TABLE VI. Calculations of Hamiltonian parameters and masses for the $c \bar{c} s q_{1} q_{2} \Sigma^{+}(1 S)$ states. All masses are in units of $\mathrm{MeV}$. The fit in the first (second) column uses values in which the corresponding $M_{0}(1 P)$ value is obtained from the first (second) column of Table $\mathrm{V}$, respectively.

\begin{tabular}{lcc}
\hline \hline$M_{0}(1 S)$ & $4258.6 \pm 1.8$ & $4255.2 \pm 1.7$ \\
$\kappa_{s c}(1 S)$ & $109.8 \pm 1.1$ & $109.8 \pm 1.1$ \\
$m_{\delta=(c s)}$ & $2069.4 \pm 10.9$ & $2069.4 \pm 10.9$ \\
$m_{\bar{\theta}}$ & $2077.2 \pm 11.6$ & $2073.5 \pm 9.0$ \\
$M_{\frac{1}{2}-}$ & $4093.9 \pm 2.4$ & $4090.5 \pm 2.3$ \\
& $4313.5 \pm 1.9$ & $4310.1 \pm 1.8$ \\
$M_{\frac{3-}{2}}$ & $4313.5 \pm 1.9$ & $4310.1 \pm 1.8$ \\
\hline \hline
\end{tabular}

\section{CONCLUSIONS}

In this paper, we have shown that the dynamical diquark model produces a spectrum of $c \bar{c} q q_{1} q_{2}$ pentaquark states that agrees very well with the four narrow $P_{c}$ resonances observed by the LHCb Collaboration. Their spectrum, as two closely spaced pairs with nearly the same mass splitting, fits neatly with the expected levels of the first excited-state multiplet $\Sigma^{+}(1 P)$ of the model, all members of which have positive parity. Specifically, $P_{c}(4312)$ and $P_{c}(4337)$ are predicted to be $\frac{1}{2}+$ states, $P_{c}(4440)$ is predicted to be a yet-unresolved $\frac{1}{2}^{+}-\frac{3}{2}^{+}$pair, and $P_{c}(4457)$ is predicted to be $\frac{3}{2}+$. Moreover, the numerical values of the Hamiltonian parameters (spin-spin, spinorbit) in this calculation are found to be closely comparable to those obtained in a study of the negative-parity hiddencharm tetraquark states $[Y(4220)$, etc.].

The two yet-unobserved states of the $\Sigma^{+}(1 P)$ multiplet are predicted to have much higher masses. The broad, opposite-parity $P_{c}(4380)$ structure does not fit into the model, and if it persists, it is much more likely a threshold effect or molecule, e.g., caused by $\bar{D} \Sigma_{c}^{*}$.

The presence of $P_{c}(4312)$ and the absence of $P_{c}(4337)$ in one production channel $\left(\Lambda_{b}\right.$ decay), and vice versa in another production channel ( $B_{s}^{0}$ decay $)$-both seen at the same experiment—is a remarkable feature. Such a pattern can be explained (if not yet fully understood) in this model by the fact that the two states are composed of the same $[\bar{c}(u d)]$ color-triplet triquark, but have distinct internal spin structures for their color-antitriplet diquark (cq) component. The heavy-quark spin content of the states, as indicated, for example, by the relative branching fractions of the resonances into $J / \psi$ or $\eta_{c}$ final states, can also serve as a useful diagnostic in uncovering the internal structure of the states.

Of exceptional importance in this analysis is the parity quantum number for all of these states. All $S$-wave molecules formed from a ground-state charmed baryon $\left(\Sigma_{c}^{+}\right.$or $\Lambda_{c}$ : parity +$)$ and a ground-state charmed meson $\left(\bar{D}^{(*)}\right.$ : parity -$)$ have negative parity. If any of the narrow $P_{c}$ states is found to have negative parity, then virtually all the analysis in this paper becomes invalidated, or at least must be radically modified.

The three states of the ground-state multiplet $\Sigma^{+}(1 S)$ in the model, which do carry negative parity, are predicted to have masses above the $J / \psi p$ threshold, but could have escaped detection by $\mathrm{LHCb}$ either due to lower statistics in the near-threshold region, or due to the states having large widths (as $S$-wave states, via their $J / \psi p$ fallapart modes).

The same model applied to the open-strange sector predicts $P_{c s}(4459)$ to be the strange analogue to the $\frac{1}{2}^{+}$ $P_{c}$ (4312). We also present predictions for all other $c \bar{c} u d s$ states in the $\Sigma^{+}(1 P)$ and $\Sigma^{+}(1 S)$ multiplets, for which the latest data gives only the vaguest indications. Future refinements of the statistics for channels like $\Xi_{b}^{-} \rightarrow$ $(J / \psi \Lambda) K^{-}$will almost certainly reveal the existence of further $P_{c s}$ resonances, and the spectrum of these new states will provide crucial information for unraveling their internal structure.

\section{ACKNOWLEDGMENTS}

This work was supported by the National Science Foundation (NSF) under Grants No. PHY-1803912 and No. PHY-2110278.
[1] R. Lebed, R. Mitchell, and E. Swanson, Prog. Part. Nucl. Phys. 93, 143 (2017).

[2] H.-X. Chen, W. Chen, X. Liu, and S.-L. Zhu, Phys. Rep. 639, 1 (2016).

[3] A. Hosaka, T. Iijima, K. Miyabayashi, Y. Sakai, and S. Yasui, Prog. Theor. Exp. Phys. 2016, 062 C01 (2016).

[4] A. Esposito, A. Pilloni, and A. Polosa, Phys. Rep. 668, 1 (2017).

[5] F.-K. Guo, C. Hanhart, U.-G. Meißner, Q. Wang, Q. Zhao, and B.-S. Zou, Rev. Mod. Phys. 90, 015004 (2018).
[6] A. Ali, J. Lange, and S. Stone, Prog. Part. Nucl. Phys. 97, 123 (2017).

[7] S. Olsen, T. Skwarnicki, and D. Zieminska, Rev. Mod. Phys. 90, 015003 (2018).

[8] M. Karliner, J. Rosner, and T. Skwarnicki, Annu. Rev. Nucl. Part. Sci. 68, 17 (2018).

[9] C.-Z. Yuan, Int. J. Mod. Phys. A 33, 1830018 (2018).

[10] Y.-R. Liu, H.-X. Chen, W. Chen, X. Liu, and S.-L. Zhu, Prog. Part. Nucl. Phys. 107, 237 (2019). 
[11] N. Brambilla, S. Eidelman, C. Hanhart, A. Nefediev, C.-P. Shen, C. Thomas, A. Vairo, and C.-Z. Yuan, Phys. Rep. 873, 1 (2020).

[12] R. Aaij et al. (LHCb Collaboration), Phys. Rev. Lett. 115, 072001 (2015).

[13] R. Aaij et al. (LHCb Collaboration), Phys. Rev. Lett. 122, 222001 (2019).

[14] R. Aaij et al. (LHCb Collaboration), arXiv:2108.04720.

[15] R. Aaij et al. (LHCb Collaboration), Sci. Bull. 66, 1278 (2021).

[16] V. Abazov et al. (D0 Collaboration), arXiv:1910.11767.

[17] J.-J. Wu, R. Molina, E. Oset, and B.-S. Zou, Phys. Rev. Lett. 105, 232001 (2010).

[18] W.-L. Wang, F. Huang, Z.-Y. Zhang, and B.-S. Zou, Phys. Rev. C 84, 015203 (2011).

[19] Z.-C. Yang, Z.-F. Sun, J. He, X. Liu, and S.-L. Zhu, Chin. Phys. C 36, 6 (2012).

[20] M. Karliner and J. Rosner, Phys. Rev. Lett. 115, 122001 (2015).

[21] X.-K. Dong, F.-K. Guo, and B.-S. Zou, Commun. Theor. Phys. 73, 125201 (2021).

[22] Z.-G. Wang, Int. J. Mod. Phys. A 36, 2150071 (2021).

[23] Z.-G. Wang, Int. J. Mod. Phys. A 35, 2050003 (2020).

[24] K. Azizi, Y. Sarac, and H. Sundu, Phys. Rev. D 103, 094033 (2021).

[25] K. Azizi, Y. Sarac, and H. Sundu, Chin. Phys. C 45, 053103 (2021).

[26] K. Azizi, Y. Sarac, and H. Sundu, Phys. Rev. D 95, 094016 (2017).

[27] H.-X. Chen, W. Chen, X. Liu, T. Steele, and S.-L. Zhu, Phys. Rev. Lett. 115, 172001 (2015).

[28] S. Brodsky, D. Hwang, and R. Lebed, Phys. Rev. Lett. 113, 112001 (2014).

[29] R. Lebed, Phys. Lett. B 749, 454 (2015).

[30] R. Lebed, Phys. Rev. D 96, 116003 (2017).

[31] J. Giron, R. Lebed, and C. Peterson, J. High Energy Phys. 05 (2019) 061.
[32] J. Giron, R. Lebed, and C. Peterson, J. High Energy Phys. 01 (2020) 124.

[33] J. Giron and R. Lebed, Phys. Rev. D 101, 074032 (2020).

[34] J. Giron and R. Lebed, Phys. Rev. D 102, 014036 (2020).

[35] J. Giron and R. Lebed, Phys. Rev. D 102, 074003 (2020).

[36] J. Giron, R. Lebed, and S. Martinez, Phys. Rev. D 104, 054001 (2021).

[37] M. Ablikim et al. (BESIII Collaboration), Phys. Rev. Lett. 126, 102001 (2021).

[38] R. Aaij et al. (LHCb Collaboration), Phys. Rev. Lett. 127, 082001 (2021).

[39] R. Zhu and C.-F. Qiao, Phys. Lett. B 756, 259 (2016).

[40] A. Ali, I. Ahmed, M. Aslam, A. Parkhomenko, and A. Rehman, J. High Energy Phys. 10 (2019) 256.

[41] P.-P. Shi, F. Huang, and W.-L. Wang, Eur. Phys. J. A 57, 237 (2021).

[42] R. Jaffe, Phys. Rep. 409, 1 (2005).

[43] A. Francis, P. de Forcrand, R. Lewis, and K. Maltman, arXiv:2106.09080.

[44] J. Gens, J. Giron, and R. Lebed, Phys. Rev. D 103, 094024 (2021).

[45] P.-P. Shi, F. Huang, and W.-L. Wang, Phys. Rev. D 103, 094038 (2021).

[46] K. Juge, J. Kuti, and C. Morningstar, Phys. Rev. Lett. 90, 161601 (2003).

[47] http://www.andrew.cmu.edu/user/cmorning/ static_potentials/SU3_4D/greet.html.

[48] S. Capitani, O. Philipsen, C. Reisinger, C. Riehl, and M. Wagner, Phys. Rev. D 99, 034502 (2019).

[49] L. Maiani, F. Piccinini, A. Polosa, and V. Riquer, Phys. Rev. D 71, 014028 (2005).

[50] R. Kleiv, T. Steele, A. Zhang, and I. Blokland, Phys. Rev. D 87, 125018 (2013).

[51] C.-W. Shen, F.-K. Guo, J.-J. Xie, and B.-S. Zou, Nucl. Phys. A954, 393 (2016).

[52] M.-L. Du, Z.-H. Guo, and J. Oller, arXiv:2109.14237. 